\title{
An investigation into the variability between different shear wave elastography systems in muscle
}

\author{
Abdulrahman M. Alfuraih ${ }^{1,2,3}$, Phil O'Connor ${ }^{3}$, Ai Lyn Tan ${ }^{2,3}$, Elizabeth M.A. Hensor ${ }^{2,3}$, \\ Paul Emery ${ }^{2,3}$, Richard J. Wakefield ${ }^{2,3}$
}

${ }^{1}$ Radiology and Medical Imaging Department, College of Applied Medical Sciences, Prince Sattam bin Abdulaziz University, Kharj, Saudi Arabia, ${ }^{2}$ Leeds Institute of Rheumatic and Musculoskeletal Medicine, Chapel Allerton Hospital, University of Leeds, United Kingdom, ${ }^{3}$ NIHR Leeds Biomedical Research Centre, Leeds Teaching Hospitals NHS Trust, Leeds, United Kingdom

\begin{abstract}
Aims: The reliability and agreement between shear wave elastography (SWE) systems using different acquisition methods in muscles is not yet established. The objectives were to determine the reliability of a new SWE system on normal resting muscles using different acquisition methods and to compare its performance to an established state-of-the-art system. Material and methods: Small, medium and large ROI sizes in addition to longitudinal, oblique and transverse orientations over five different locations within the rectus femoris muscle were tested using the new system. Results were compared to the established system to test for inter-system reproducibility. Results: Lowest within-subject coefficient of variance (4.3\%) and shear wave velocity $(1.83 \mathrm{~m} / \mathrm{s})$ were associated with the medium ROI and longitudinal orientation from the lateral location. This combination resulted in a strong internal agreement of intra-class correlation of $0.76(0.57-0.89)$ for the new system and an almost perfect agreement of $0.92(0.82-0.97)$ for the established. Inter-system reproducibility for the best combination was $0.71(0.48-1)$ with a mean velocity difference $\pm 95 \%$ limits of agreement of $0.07 \pm 0.49 \mathrm{~m} / \mathrm{s}$. Conclusions: Altering SWE acquisition methods can produce variable results. The new system produced reliable results that are comparable with but not as reliable as the established.
\end{abstract}

Keywords: elastography; ultrasound; muscles; reliability

\section{Introduction}

The mechanical properties of muscles can be altered by a range of pathologies, such as muscle dystrophy [1], spasticity [2], and inflammation [3]. Muscle stretch and contraction elastic elements are major determinants of muscle function and efficient locomotion [4,5]. The measurement of these parameters has largely been explored using myotonometry but this has not been shown to have acceptable accuracy and is limited to superfi-

Received 28.05.2017 Accepted 26.07.2017

Med Ultrason

2017, Vol. 19, No 4, 392-400

Corresponding author: Abdulrahman M. Alfuraih

LIRMM, $2^{\text {nd }}$ Floor,

Chapel Allerton Hospital,

Chapeltown Road, Leeds, LS7 4SA, UK.

Phone: +44 1133924854

E-mail:mt14ama@leeds.ac.uk cial muscles only [6,7]. Additional techniques such as magnetic resonance elastography (MRE) and standard ultrasonography can assess the mechanical properties of tissues in-vivo. However, these techniques have several drawbacks, for instance, the high cost and poor temporal resolution of MRE and the indirect stiffness estimations of standard ultrasonography $[8,9]$. New methods have been sought to improve this aspect of muscle assessment.

Shear wave elastography (SWE) is one of the latest ultrasound-based elastography techniques. It provides quantitative tissue stiffness assessment by measuring shear wave velocity. In SWE, Acoustic Radiation Force Impulse (ARFI) produced by a transducer induces tissue displacement, which, in turn, causes a propagation of transverse shear waves within that tissue. The velocity of these shear waves is directly related to tissue shear modulus. That is, higher velocities equate to higher stiff- 
ness and vice versa. Currently, there are numerous commercially available SWE systems. Each uses a different patent-protected technology, image processing and algorithms to quantify tissues elasticity. Research comparing reproducibility of these systems in muscle SWE is scarce.

Elastography has been well validated for imaging breast and liver tissue [10,11]. Its application for the investigation of musculoskeletal conditions is not so well established. Muscles, unlike most visceral organs, are inherently anisotropic in nature. They are heterogenic structures with variable aponeurotic anatomy, fiber lengths and pennation angles. In addition, their length and shape are affected by passive and active movements. Therefore, in-vivo stiffness assessment of muscles is complex because of its varying anatomical structure.

A review of the literature demonstrates some variability regarding probe orientation. Most studies have focused on the longitudinal plane with others favoring the transverse [12-15]. Several studies have investigated the effect of scanning muscles in more than one plane [1618] with most reporting better reliability in the longitudinal orientation. Only one study was found that evaluated potential SWE differences from different locations within a muscle and found differences in elasticity and reading reliability [18]. Several SWE manufacturers offer the option to select an ROI of various sizes and shapes. Two studies have tested the use of variable ROI sizes and reported conflicting results; it is not clear whether ROI size has no effect [14] or whether variability is greater with smaller ROI [19]

Acquisition method standardization will help reduce technically-induced variation and improve our ability to evaluate the sensitivity to change of muscle elastography to interventions. Additionally, knowledge of muscle SWE reproducibility between systems is vital for results comparison in future studies. To our knowledge, no studies have investigated muscle SWE performance between different manufacturers, which might be attributed to the dominance of older systems over newly introduced ones.

This study aimed to determine how different technical and acquisition variables might influence stiffness calculations in normal muscle and whether they have a greater effect on some systems more significantly than others. The specific objectives were threefold: first, to test the effect of using different probe orientations, locations and ROI size combinations; second, to determine the reliability of a relatively new SWE system in normal human resting muscles and finally, to compare the new system performance to another well-validated and established SWE system.

\section{Materials and methods}

\section{Subjects}

Twenty healthy volunteers (13 males and 7 females) of mean [standard deviation (SD)] age 38.7 (14.9) years and body mass index (BMI) $25.3(4.2) \mathrm{kg} / \mathrm{m}^{2}$ participated. Volunteers with a history of musculoskeletal or rheumatic conditions were excluded. All subjects were asked to refrain from any strenuous or sporting activities 24 hours prior to the study to eliminate any possible confounding exercise effect. The study had the Research Ethics Committee approval and was performed in accordance with Good Clinical Practice guidelines. All subjects gave written consent.

\section{Equipment}

Two-dimensional SWE measurements were acquired using the recent commercially released SWE package for the General Electric LOGIQ E9 system [(LE9); GE Healthcare, Buckinghamshire, UK] using a linear 9-5 $\mathrm{MHz}$ probe. The LE9 utilizes the comb-push excitation method that simultaneously transmits multiple comb-like ARFI pulses that produce shear waves. Then, time-interleaved shear wave tracking is used to detect shear waves and estimate shear wave velocity [20].

A rectangular shear wave box of $2 / 2.2 \mathrm{~cm}$ was used. The box was centered at a depth of $2.5 \mathrm{~cm}$, although this varied slightly between patients due to varying body habitus. Additionally, an SWE scale of $0-7.1 \mathrm{~m} / \mathrm{s}$ was chosen. Because of the anisotropic nature of muscles, and to the Youngs' Modulus (in $\mathrm{kPa}$ ) assumptions of isotropy and homogeneity, measurements were recorded in units of shear wave velocity $(\mathrm{m} / \mathrm{s})$. To avoid probe-induced stiffness, minimal sufficient probe compression was used to ensure good skin contact.

\section{Shear Wave Elastography feasibility and reliability}

A board-certified sonographer (AMA) with more than four years' scanning experience performed the SWE measurements. To test the effect of aponeurotic structures and angle of muscle fibers on SWE readings, the rectus femoris muscle was chosen because of its bipennate anatomy. Subjects were asked to lie supine in a comfortable position. Additionally, before acquiring measurements, subjects rested on the table for five minutes in order to avoid any potential locomotion-induced stiffness. Any SWE acquisition containing stripy or marked artefacts were excluded. Three variables were tested in this study:

1. Location. Using ultrasound guidance, the proximal and distal myotendinous junctions of the right leg were marked on the volunteer using a surgical marker. The muscle was then marked at $25 \%$ (superior), $50 \%$ (mid) and $75 \%$ (inferior) to define reference points for the level and location of acquisitions (fig 1a). The central aponeu- 
394 Abdulrahman M. Alfuraih et al An investigation into the variability between different shear wave elastography systems in muscle

rotic line of the rectus femoris muscle served as a reference to the locations between the origin and insertion. Two additional points were marked on either side of the mid-point (medial and lateral). Superior, mid and inferior points were performed to test muscle aponeurosis effect on SWE. Medial and lateral points ensured scanning was performed in the center of both muscle bellies.

2. Orientation. The probe was positioned in three different angles according to fiber orientation: transverse (across the fibers; fig 1b1), longitudinal (along the fibers; fig1b2) and oblique (along the thigh; fig 1b3). This variable tests the effect of muscle anisotropy on SWE. For the superior, mid and distal locations, where the two rectus femoris bellies merge to the central aponeurosis, the probe was oriented across the thigh for a transverse plane and along the aponeurosis for the oblique fiber orientation. As for the longitudinal plane, the probe was rotated slightly medially or laterally until multiple fibers could be visualized on the B-mode image.

3. ROI size. Three ROI sizes $15 \mathrm{~mm}^{2}$ (small), $75 \mathrm{~mm}^{2}$ (medium) and $200 \mathrm{~mm}^{2}$ (large) were selected within each SWE acquisition. The area was displayed to ensure accurate and consistent ROI sizes (fig 2). The circular ROI for the different sizes were always located at the center of the shear wave box. Readings were repeated three times for each combination of location-orientation-ROI. The probe was removed and replaced each time. As a result, each examination consisted of $135 \mathrm{SWE}$ readings (five sites, three probe orientations, three ROI sizes and three repetitions).

\section{Shear Wave Elastography system comparison}

Upon finishing the LE9 SWE reliability scans, subjects were moved to an adjacent room to have the examinations repeated on another SWE system machine [(AIX); Aixplorer, Supersonic Imagine, Aix-enProvence, France]. To generate shear waves, the AIX system sends multiple successive ARFI which are focused at different depths in tissue. These pulses generate a "Mach cone" shaped shear wave profile, which increases the efficiency and amplifies the distance of the propagated shear waves [21]. Following this, utilizing ultrafast imaging technology, a flat wave insonifies the whole imaging plane to track and quantify shear wave velocity. Thirteen participants were tested and each rested in a supine position as previous examinations. Readings were obtained using the SuperLinearTM SL10-2MHz probe. As near as possible probe frequency, shear box size and depth, scale, SWE mode and reference points were applied. Due to time constraints, the full study protocol was shortened with only mid, lateral, and medial locations being scanned. Additionally, only the medium ROI size of $10 \mathrm{~mm}$ in diameter was used, which is equivalent to 75

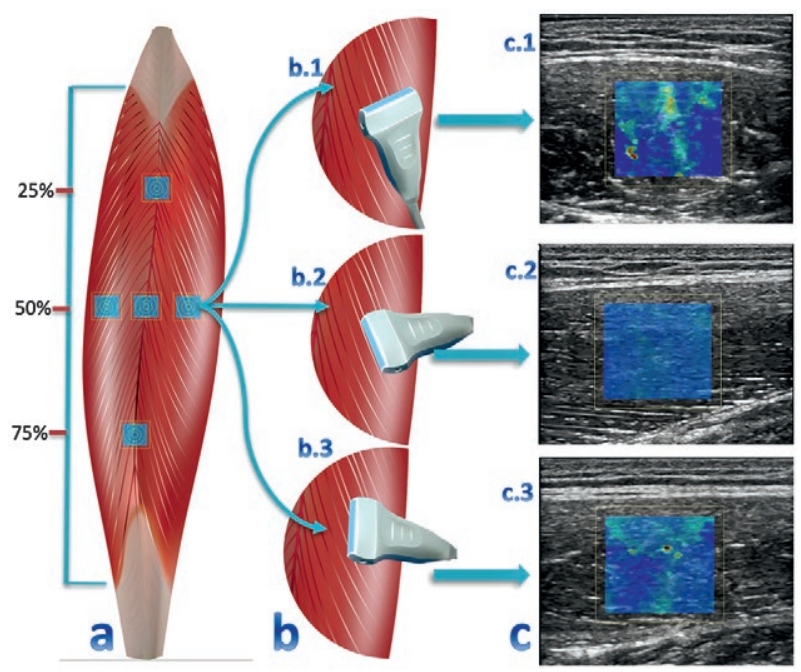

Fig 1. The scanning protocol: "a" shows the rectus femoris muscle with the three points used between the proximal and distal myotendinous junctions in addition to the five locations selected within; "b" demonstrates the three probe orientations of the medial location as an example: b.1 transverse (across the fibres), b.2 longitudinal (along the fibres), and b.3 oblique (along the thigh axis); "c" shows each orientation's corresponding image.

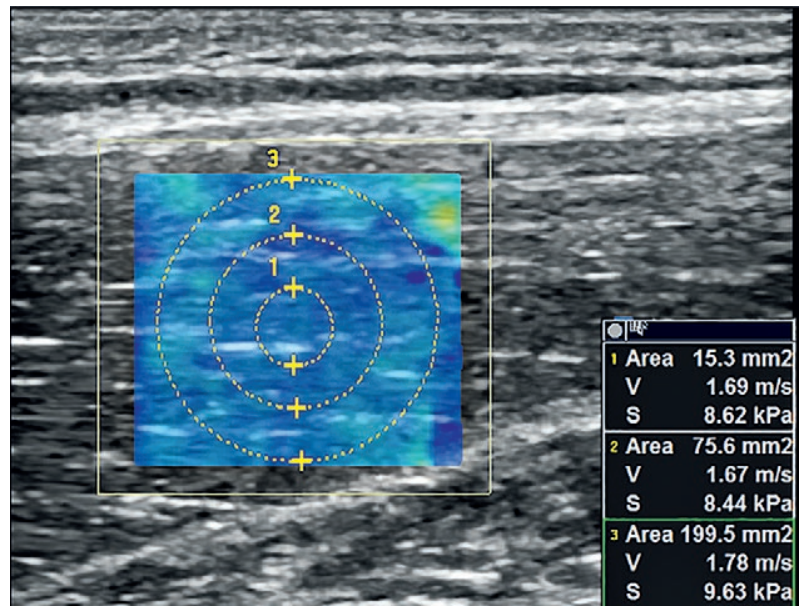

Fig 2. The three ROI sizes: small (1), medium (2) and large (3) with corresponding SWE readings. The image was acquired longitudinally from the lateral location.

$\mathrm{mm}^{2}$ (medium) on LE9. Each comparative examination consisted of 27 SWE readings (three sites, three probe orientations, one ROI sizes and three repetitions).

\section{Statistical analysis}

Statistical analyses were performed using SPSS V.21 (IBM Corp. Armonk, NY). Because within-subject variability was found to be related to the magnitude of measurement, measurement error was represented as within-subject coefficient of variation (WSCV). We 
first obtained the within-subject standard deviation (WSSD) of log-transformed values [22], then calculated $\mathrm{WSCV}=\exp (\mathrm{WSSD})-1$ [23]. Reliability and variability of different ROI sizes were assessed in a single, commonly-used plane (longitudinal) and location (lateral), then one ROI size was chosen to simplify the reliability assessments in each combination of different planes and locations.

Intra-class correlation coefficients (ICC) were used to measure LE9 intra-system reliability, and were interpreted as follows: $0.00-0.20$ 'poor agreement', 0.21-0.40 'fair agreement', 0.41-0.60 'moderate agreement', 0.61-0.80 'substantial agreement' and $>0.80$ 'almost perfect agreement' [24]. $\mathrm{ICC}_{(1,1)}$ was used for intra-system reliability, whilst $\mathrm{ICC}_{(3,1)}$ was used for inter-system reliability; it was assumed that a single acquisition rather than a mean of repeated acquisitions would be used in clinical practice.

To compare LE9 with AIX, ICCs for inter-system reproducibility were calculated to include each of the three repeated measurements available [25]. Additionally, to assess inter-system agreement Bland-Altman 95\% limits of agreement (LOA) and their $95 \%$ confidence intervals were computed. This involved taking an initial mean of the three measurements per condition, then adjusting the variance estimates for the inter-system differences accordingly [26].

\section{Results}

\section{Location, orientation and ROI effects}

Velocities did not differ substantively by ROI size; reliability indices indicated the small ROI did not perform quite as well as the larger sizes (Table I). Medium ROI demonstrated the best reliability and lowest WSCV and was chosen for the remaining analyses.

Using medium ROI size, mean velocities were generally lowest in the longitudinal plane and highest in the transverse plane, irrespective of location (fig 3). In the oblique plane, values were higher and more variable than in the longitudinal plane, but showed a similar pattern across locations: velocities in both planes were highest in the superior location, and lowest in lateral/medial. In contrast, the transverse plane velocity readings were less affected by locations, except for inferior, which resulted in a significantly higher mean velocity.

Reliability of LE9 measurements

WSCV decreased gradually from transverse, to oblique, to longitudinal; it was highest in the transverse plane and medial location (18.0\%) and lowest in the longitudinal plane and lateral location (4.3\%) (fig 4, Table II). Transverse planes generally resulted in lower ICCs indicating 'fair' agreement, except for the superior and lateral locations (Table III). There was greater reliability across all longitudinal planes. The inferior-longitudinal and lateral-longitudinal combinations resulted in the highest ICCs of 0.80 and 0.76 respectively, which represent a substantial agreement. Oblique plane results showed moderate agreement in almost all locations. Overall, the trends with respect to the combinations that

Table I. Shear wave velocities for the three ROI sizes from the lateral location using the longitudinal orientation.

\begin{tabular}{|c|c|c|c|}
\hline \multirow{2}{*}{$\begin{array}{l}\text { ROI } \\
\text { size }\end{array}$} & Velocity $(\mathrm{m} / \mathrm{s})$ & \multirow{2}{*}{$\begin{array}{l}\text { WSCV } \\
(\%)\end{array}$} & \multirow[t]{2}{*}{ ICC (95\% CI) } \\
\hline & Geo. mean $(95 \%$ CI) & & \\
\hline Small & $1.86(1.78-1.94)$ & 6.8 & $0.61(0.37-0.80)$ \\
\hline Medium & $1.83(1.77-1.90)$ & 4.3 & $0.76(0.57-0.89)$ \\
\hline Large & $1.86(1.79-1.93)$ & 4.3 & $0.74(0.54-0.87)$ \\
\hline
\end{tabular}

Geo. mean - Geometric mean, Geometric mean velocity and $95 \%$ Confidence Intervals (CI) were calculated using the mean of the three repeated measurements per subject.

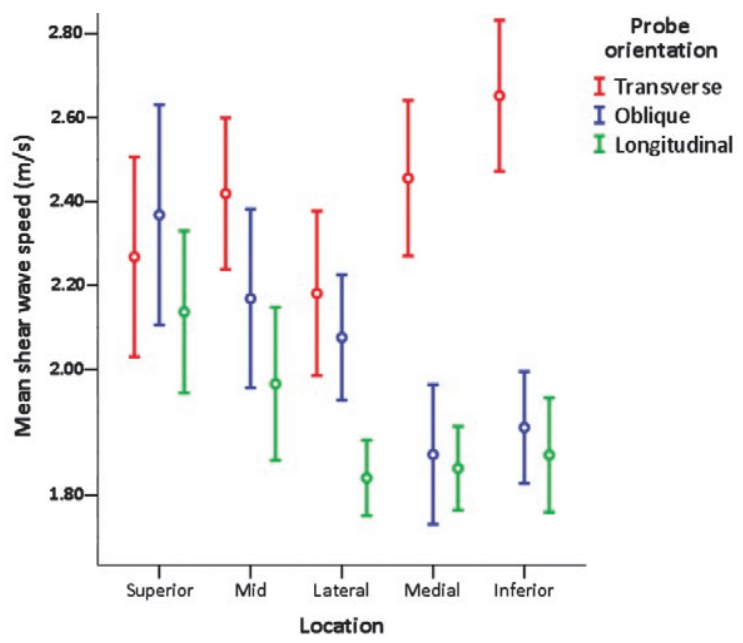

Fig 3. Mean (ln-transformed) geometric mean shear wave velocity and $(95 \% \mathrm{CI})$ recorded for each method using medium ROI.

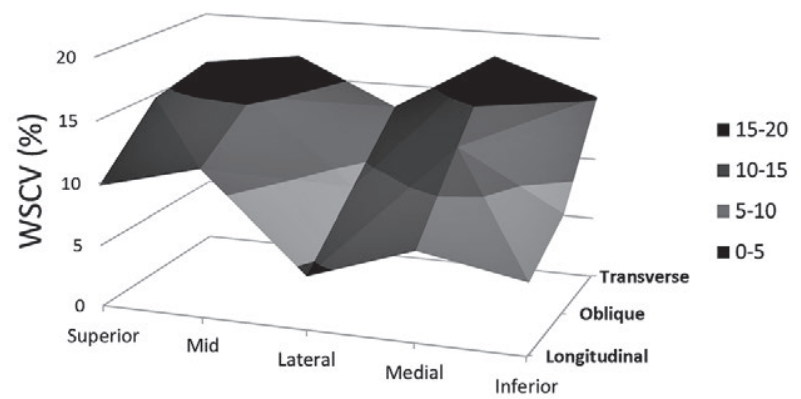

Fig 4. Surface plot showing within-subject coefficient of variation (WSCV) for each method using medium ROI. The longitudinal-lateral combination achieved the lowest variability $(4.3 \%)$. 
396 Abdulrahman M. Alfuraih et al An investigation into the variability between different shear wave elastography systems in muscle

Table II. Shear wave velocities means, lower and upper 95\% confidence intervals and within-subjects coefficient of variance for all combinations of orientation and location.

\begin{tabular}{lllll}
\hline Orientation & Location & ROI size & Velocity (m/s) & WSCV (\%) \\
\cline { 3 - 4 } & & & Geo. mean (95\% CI) & 15.8 \\
\hline Transverse & Superior & Medium & $2.26(2.06-2.49)$ & 16.9 \\
& Mid & Medium & $2.40(2.24-2.58)$ & 13.1 \\
& Lateral & Medium & $2.19(2.02-2.37)$ & 18.0 \\
Medial & Medium & $2.44(2.27-2.63)$ & 15.2 \\
Oblique & Inferior & Medium & $2.64(2.46-2.84)$ & 14.7 \\
& Superior & Medium & $2.36(2.12-2.62)$ & 15.4 \\
& Mid & Medium & $2.18(2.00-2.37)$ & 12.7 \\
Lateral & Medium & $2.10(1.98-2.23)$ & 8.1 \\
& Medial & Medium & $1.88(1.75-2.00)$ & 9.8 \\
& Inferior & Medium & $1.92(1.82-2.03)$ & 11.9 \\
& Superior & Medium & $2.15(1.99-2.32)$ & 4.3 \\
& Mid & Medium & $2.01(1.86-2.16)$ & 7.2 \\
& Lateral & Medium & $1.83(1.77-1.90)$ & 5.7 \\
\hline
\end{tabular}

Geo. Mean - Geometric mean; ROI - region of interest; WSCV- within-subject coefficient of variation. Geometric mean velocity and 95\% $\mathrm{CI}$ were calculated using the mean of the three repeated measurements per patient.

Table III. Reliability of different location and orientation combinations demonstrated as intraclass correlation coifficient (ICC) within each (intra) and between (inter) systems. The mean difference is also listed with associated limits of agreement (LOA).

\begin{tabular}{|c|c|c|c|c|c|c|}
\hline \multirow[t]{2}{*}{ Orientation } & \multirow[t]{2}{*}{ Location } & \multicolumn{2}{|c|}{ Intra-system ICC $(95 \%$ CI) } & \multirow{2}{*}{$\begin{array}{l}\text { Inter-system ICC } \\
(95 \% \text { CI })^{*}\end{array}$} & \multirow{2}{*}{$\begin{array}{l}\text { Mean } \\
\text { difference }(\mathrm{m} / \mathrm{s})\end{array}$} & \multirow[t]{2}{*}{ LOA $(95 \%$ CI $)$} \\
\hline & & LE9 & AIX & & & \\
\hline \multirow[t]{5}{*}{ Transverse } & Superior & $0.64(0.41-0.82)$ & - & - & - & - \\
\hline & Mid & $0.47(0.20-0.72)$ & $0.81(0.61-0.93)$ & $0.12(-0.24-1)$ & 0.18 & $\pm 1.15(0.66-1.65)$ \\
\hline & Lateral & $0.62(0.38-0.81)$ & $0.87(0.71-0.95)$ & $0.65(0.41-1)$ & 0.17 & $\pm 0.64(0.40-0.88)$ \\
\hline & Medial & $0.41(0.14-0.68)$ & $0.81(0.59-0.93)$ & $0.27(-0.03-1)$ & 0.19 & $\pm 0.95(0.58-1.32)$ \\
\hline & Inferior & $0.48(0.21-0.72)$ & - & - & - & - \\
\hline \multirow[t]{5}{*}{ Oblique } & Superior & $0.72(0.51-0.87)$ & - & - & - & - \\
\hline & Mid & $0.61(0.37-0.81)$ & $0.95(0.88-0.98)$ & $0.15(-0.21-1)$ & -0.10 & $\pm 1.06(0.60-1.51)$ \\
\hline & Lateral & $0.65(0.42-0.83)$ & $0.70(0.42-0.88)$ & $0.53(0.26-1)$ & 0.08 & $\pm 0.54(0.34-0.74)$ \\
\hline & Medial & $0.45(0.18-0.70)$ & $0.90(0.78-0.97)$ & $0.60(0.30-1)$ & -0.03 & $\pm 0.45(0.26-0.64)$ \\
\hline & Inferior & $0.62(0.38-0.81)$ & - & - & - & - \\
\hline \multirow[t]{5}{*}{ Longitudinal } & Superior & $0.71(0.50-0.86)$ & - & - & - & - \\
\hline & Mid & $0.65(0.41-0.82)$ & $0.92(0.82-0.97)$ & $0.43(0.18-1)$ & 0.12 & $\pm 0.56(0.34-0.78)$ \\
\hline & Lateral & $0.76(0.57-0.89)$ & $0.98(0.95-0.99)$ & $0.71(0.48-1)$ & 0.07 & $\pm 0.49(0.31-0.68)$ \\
\hline & Medial & $0.55(0.29-0.77)$ & $0.97(0.92-0.99)$ & $0.54(0.27-1)$ & 0.05 & $\pm 0.38(0.23-0.53)$ \\
\hline & Inferior & $0.80(0.64-0.91)$ & - & - & - & - \\
\hline
\end{tabular}

* One-sided lower limit 95\% confidence interval (CI).

yielded the lowest variability were similar, whether they were assessed using the ICC values, or the WSCVs.

\section{System comparison}

SWE on AIX produced almost perfect intra-system reliability $(>0.8)$ in all combinations except lateraloblique, in comparison to the moderate-substantial internal consistency found for LE9 (Table III). Similar to LE9, the lateral-longitudinal combination produced the most internally consistent SWE readings.

Comparing the two systems, transverse planes and mid locations were associated with poor to fair inter-sys- tem ICCs. The longitudinal-lateral combination achieved the strongest agreement of ICC $(95 \% \mathrm{CI})=0.71(0.48$, 1). Figure 5 shows that both systems recorded high mean readings in the transverse plane; however, the CIs were wide in the longitudinal plane. In longitudinal, both recorded lower and more stable readings. As for mean differences, the best results were obtained in the medial and lateral locations in oblique or longitudinal planes; mean differences ranged -0.03 to +0.08 indicating little bias between the systems for these combinations. However, the $95 \% \mathrm{LOA}$ and the confidence intervals around them 
were comparatively wide, ranging from \pm 0.38 ( 0.23 , $0.53)$ to $\pm 0.54(0.34,0.74)$, given that mean velocities were approximately $1.80 \mathrm{~m} / \mathrm{s}$. Bias was greatest using the transverse plane in the medial and mid locations; velocities from LE9 exceeded AIX by 0.19 and $0.18 \mathrm{~m} / \mathrm{s}$ respectively and the limits of agreement were twice as wide as for lateral-longitudinal. Bland-Altman plots for examples of the best (lateral-longitudinal) and worst (mid-transverse) combinations are presented in Figure 6. The scatterplot in Figure 7, which combines observations from the different locations, suggests that although no trend was visible for individual location-plane combinations, when considered together disagreement seemed to increase in measurements at higher velocities. However, this could be because most of the higher velocities were recorded in the transverse plane.

\section{Discussions}

SWE is a promising modality that has demonstrated its value in several medical specialties. Currently, relatively little data exists on muscle and, specifically, how different acquisition methods and machines affect elasticity readings.

The first objective of this study was to evaluate the influence of using various ROI sizes, probe orientations and locations within the rectus femoris muscle. With respect to the ROI size, in a previous study it was assumed that the mean ROI velocity is constant throughout the muscle [27]. Our results for ROI size were similar to those of Ates et al [19] in that a smaller ROI is associated with less reliable readings. Kot et al [14] found no significant difference in the mean elasticity values between small, medium, and large ROI sizes; however, this inferential statistical approach of testing for the difference would not detect changes in variability between the different sizes. Our results demonstrate that using a small ROI produced greater variability and hence a lower ICC than a medium or large ROI. This finding should be interpreted cautiously because the ROI size might be tailored to the specific purpose of the examination. For example, a large ROI may be necessary for diseases affecting a large muscle area and a small ROI may be more suitable for small focal lesions. This finding may accord some superiority to two-dimensional SWE systems that allow the free selection of ROI size over point SWE systems that have a fixed, predefined ROI size.

Our results suggest that a longitudinal (parallel to muscle fibers) orientation is the best probe orientation to use when scanning muscles. When defining to the probe orientation in anisotropic structures, the orientation of the fibers must be referred to, and not the direction of

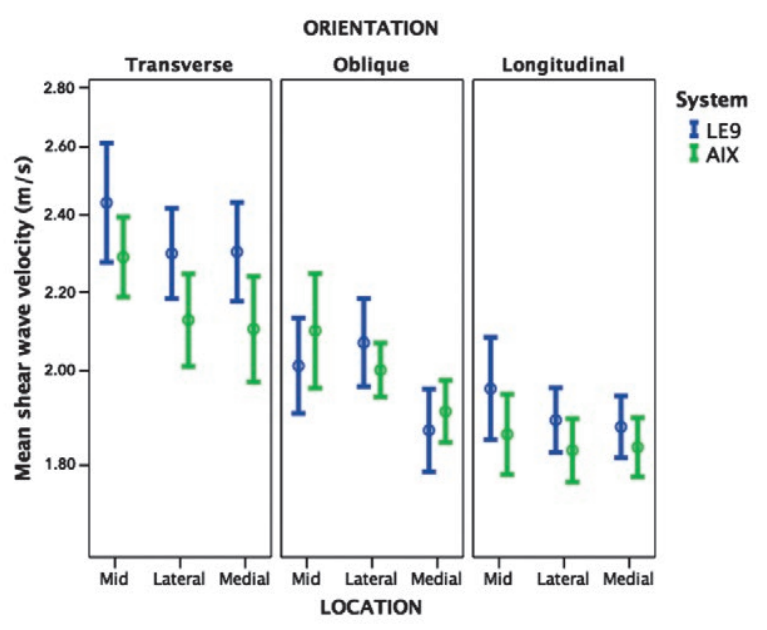

Fig 5. Error bars representing mean (ln transformed) shear wave velocity and $95 \%$ CI between the two systems for each method using medium ROI.
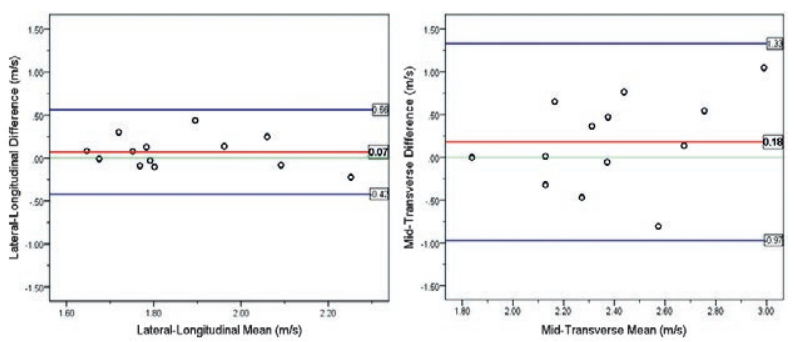

Fig 6. Two Bland-Altman plots of LE9 and AIX SWE estimations for the best (left: lateral longitudinal) and worst agreement (right: mid transverse). The middle green line is the zero-difference line, the red line is the mean difference between the two measurements and the two blue lines represent the upper and lower $95 \%$ limits of agreement.

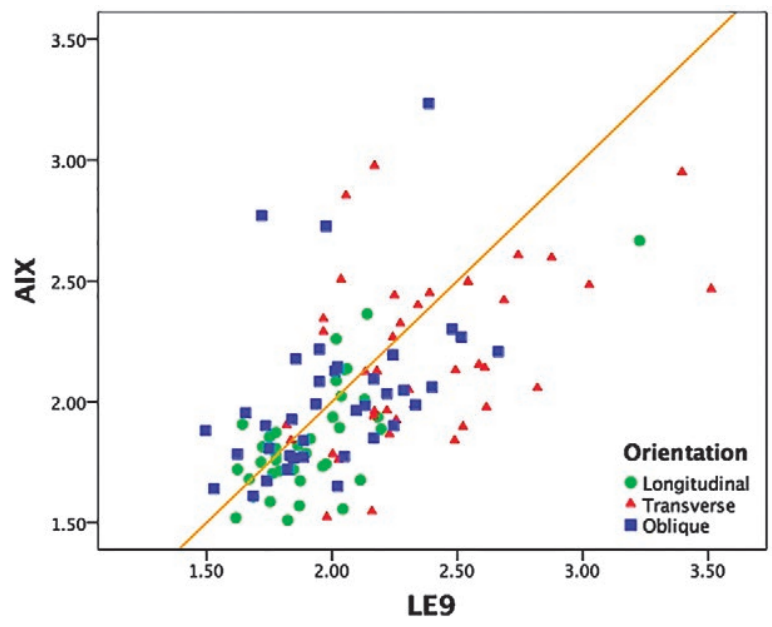

Fig 7. Scatterplot of the association between the two systems. The orange line represents ideal agreement $(\mathrm{y}=1 * \mathrm{x})$. 
the muscle or body part. This is particularly important in muscles with fibers running oblique to the longitudinal axis of the muscle such as the rectus femoris. Therefore, studies that merely report the probe being oriented 'longitudinally' might actually have been orientated at an oblique plane relative to the muscle fibers $[18,28]$. As for mean velocities, our findings are in contrast to those of Gennisson et al [16] and Cortez et al [18], who both observed slower velocities in the transverse plane. The disagreement could be related to the different muscle groups investigated. Moreover, in a relaxed unloaded muscle, the shear wave velocity is slower in the longitudinal plane than in the transverse, but as the muscle is loaded, the velocity in the longitudinal plane increases and exceeds the transverse [29]. Nevertheless, our results are in agreement with those reported for the rectus femoris muscle by Carpenter et al [30]. We found that both systems were affected by anisotropy in the transverse and oblique planes to the same extent, as velocities increased and became less stable. When qualitatively assessing SWE boxes, longitudinal planes were associated with a homogenous low SWE stiffness map. In comparison, the heterogeneous high SWE stiffness maps observed in the transverse planes may be induced due to the poor propagation of shear waves in an anisotropic structure. Oblique plane maps produced a lower level of heterogeneity than transverse plane maps, but these were not as uniform as longitudinal plane maps. This finding was observed in both systems.

With regard to the probe location within the muscle, lateral and medial regions showed the least variable readings. This shows the importance of standardizing the site of acquisition within muscles. A previous study also found differences; however, the locations selected did not include aponeurotic structures [18]. Based on the evidently increased variability at the superior, mid and inferior sites, we support the avoidance of locations where tendon, myotendinous, and myoaponeurotic structures are present in the muscular region being evaluated by SWE. Since ICCs can vary between homogeneous and heterogeneous populations, even in cases in which the within-patient variability remains the same, some authors advocate the reporting of WSCVs instead. We have provided both sets of figures and found the trends with respect to the location-plane combinations yielding the least variable results to be similar.

The second objective evaluated the reliability of the LE9 in vivo. To our knowledge, this is the first study to test this system's reliability and compare its performance on musculoskeletal imaging. Here, we assessed LE9 reliability on the rectus femoris muscle in a resting relaxed position. This was done by testing the intra-system repro- ducibility as shown by the ICC in Table III. Briefly, this test compared the agreement between the three repeated acquisitions for each location and orientation combination. The effects of location and orientation apparent from our agreement analysis were similar to that reported by Cortez et al [18]. The poor agreement observed in transverse planes might result from the anisotropic nature of muscles that might have disturbed shear wave propagation. On the other hand, scanning parallel to muscle fibers yielded the highest agreement. These results show that LE9 can reproduce longitudinal plane acquisitions better than transverse and oblique planes in all locations. The best reliability was achieved in the lateral location and longitudinal probe angle with substantial agreement.

The final objective was to compare the LE9 SWE performance to an established shear wave unit (AIX). To our knowledge, no previous studies have tested the intermachine reproducibility between different SWE systems in muscle. Each system uses a patent-protected technology that is fundamentally different in terms of shear wave generation and tracking. The comb-push excitation method of LE9 sends multiple smaller laterally-separated push pulses simultaneously. This method may produce shear waves of lower amplitude when compared to the focused Mach cone push method of AIX. This fundamental difference may suggest that AIX performs better on focal depths deeper than $2.5 \mathrm{~cm}$ due to its superior focusing technique and higher shear wave amplitude. However, investigating focal depths is not within the scope of our study. AIX achieved almost perfect consistency ranging from 0.81 to 0.97 , which is relatively similar to the 0.83 reported by Lacourpaille et al [31]. Furthermore, others found higher ICCs of 0.98 and 1 when testing AIX on different muscles [17,19]. These higher AIX agreements could potentially have been enhanced by not removing and reapplying the probe between acquisitions. In certain location/plane combinations, LE9 performed comparably to the AIX system. The transverse plane had a greater effect on intra-system ICCs for LE9 than AIX, resulting in comparatively poor inter-system ICCs. The lower amplitude, less-focused comb-push method of LE9 may have been challenged in this anisotropic orientation, as explained by the decreased intra-system reliability in the transverse plane. Only one study was found that compared LE9 and AIX performances. It was conducted on a phantom in vitro experiment and single samples of breast and liver in vivo cases. As we found in the current study, there was good agreement with AIX performing slightly better [32]. The current, the most feasible and reliable combination (longitudinal-lateral) resulted in a substantial agreement between the two systems according to ICC measures. The Bland-Altman plots shown in 
Figure 4 demonstrate the importance of selecting optimum acquisition techniques to obtain reproducible SWE estimations between different systems. Despite the high ICC we obtained, the $95 \%$ LOA were wide even for the best-performing longitudinal-lateral combination $( \pm 0.49$ $\mathrm{m} / \mathrm{s}$ ), which indicates a potential for disagreement between measurements of approximately $26 \%$ relative to the mean. However, there were also wide confidence intervals around the LOA; a larger study (around $\mathrm{n}=100$ ) focusing on this single location-orientation combination would be required to obtain more accurate estimates of these limits. This would warrant future studies on muscle SWE wishing to compare results between different systems.

Some limitations might be raised regarding this study. First, only one sonographer performed all the acquisitions and consequently, inter-operator reproducibility was not tested. Second, the sample size was relatively small; to compensate for this, the acquisition protocols used allowed a large number of acquisitions. However, further work would be needed to fully evaluate the $95 \%$ limits of agreement with the AIX system. Third, the longitudinal probe orientation at the superior, mid, and inferior locations was problematic because of the unique rectus femoris pennation anatomy, which might have resulted in a slight deviation towards medial or lateral muscle bellies.

\section{Conclusions}

The results obtained reveal that using different ROI sizes, locations and probe orientations can individually and collectively produce variable SWE estimations. SWE systems demonstrated strongest internal agreement when the probe was in a longitudinal plane relative to the muscle fibers in a predefined area that does not contain myotendinous or myoaponeurotic structures, and when using a medium to large ROI size $\left(>75 \mathrm{~mm}^{2}\right)$. Consideration of these acquisition factors in clinical practice could help yield reliable and more reproducible readings. Using these acquisition methods resulted in the highest internal agreement for both systems. However, when comparing the mean shear wave velocity between the two systems, the $95 \%$ limits of agreement were wide and results could vary by $26 \%$. Therefore, studies using different SWE systems should be compared with care and prospective studies should use the same machine. Future research studies should investigate inter-reproducibility of other systems to confirm these study findings.

\section{Acknowledgments}

The research is supported by the National Institute for Health Research (NIHR) Leeds Musculoskeletal
Biomedical Research Centre (LMBRC). The views expressed are those of the author(s) and not necessarily those of the NHS, the NIHR or the Department of Health. The authors would like to thank Supersonic Imagine for the short-term loan of their machine.

\section{Conflict of interest: none}

\section{References}

1. Cornu C, Goubel F, Fardeau M. Muscle and joint elastic properties during elbow flexion in Duchenne muscular dystrophy. J Physiol 2001;533:605-616.

2. Fridén J, Lieber RL. Spastic muscle cells are shorter and stiffer than normal cells. Muscle Nerve 2003;27:157-164.

3. Song Y, Lee S, Yoo DH, Jang KS, Bae J. Strain sonoelastography of inflammatory myopathies: comparison with clinical examination, magnetic resonance imaging and pathologic findings. Br J Radiol 2016;89:20160283.

4. Roberts TJ. Contribution of elastic tissues to the mechanics and energetics of muscle function during movement. J Exp Biol 2016;219:266-275.

5. Ettema G. Effects of contraction history on control and stability in explosive actions. J Electromyogr Kinesiol 2002;12:455-461.

6. Korhonen R, Vain A, Vanninen E, Viir R, Jurvelin JS. Can mechanical myotonometry or electromyography be used for the prediction of intramuscular pressure? Physiol Meas 2005;26:951-963.

7. Chuang LL, Wu CY, Lin KC. Reliability, validity, and responsiveness of myotonometric measurement of muscle tone, elasticity, and stiffness in patients with stroke. Arch Phys Med Rehabil 2012;93:532-540.

8. Hong SH, Hong SJ, Yoon JS, et al. Magnetic resonance elastography (MRE) for measurement of muscle stiffness of the shoulder: feasibility with a 3 T MRI system. Acta Radiol 2016;57:100-106.

9. Kubo K, Kanehisa H, Fukunaga T. Gender differences in the viscoelastic properties of tendon structures. Eur J Appl Physiol 2003;88:520-526.

10. Barr RG, Nakashima K, Amy D, et al. WFUMB guidelines and recommendations for clinical use of ultrasound elastography: Part 2: breast. Ultrasound Med Biol 2015;41:11481160.

11. Ferraioli G, Filice C, Castera L, et al. WFUMB guidelines and recommendations for clinical use of ultrasound elastography: Part 3: liver. Ultrasound Med Biol 2015;41:11611179.

12. Ewertsen C, Carlsen JF, Christiansen IR, Jensen JA, Nielsen MB. Evaluation of healthy muscle tissue by strain and shear wave elastography-Dependency on depth and ROI position in relation to underlying bone. Ultrasonics 2016;71:127133.

13. Rosskopf AB, Ehrmann C, Buck FM, Gerber C, Flück M, Pfirmann CW. Quantitative Shear-Wave US Elastography of the Supraspinatus Muscle: Reliability of the Method and 
Relation to Tendon Integrity and Muscle Quality. Radiology 2015;278:465-474.

14. Kot BC, Zhang ZJ, Lee AW, Leung VY, Fu SN. Elastic modulus of muscle and tendon with shear wave ultrasound elastography: variations with different technical settings. PLoS One 2012;7:e44348.

15. Akagi R, Takahashi H. Acute effect of static stretching on hardness of the gastrocnemius muscle. Med Sci Sports Exerc 2013;45:1348-1354.

16. Gennisson JL, Deffieux T, Macé E, Montaldo G, Fink M, Tanter M. Viscoelastic and anisotropic mechanical properties of in vivo muscle tissue assessed by supersonic shear imaging. Ultrasound Med Biol 2010;36:789-801.

17. Miyamoto N, Hirata K, Kanehisa H, Yoshitake Y. Validity of measurement of shear modulus by ultrasound shear wave elastography in human pennate muscle. PLoS One 2015; 10:e 0124311.

18. Cortez CD, Hermitte L, Ramain A, Mesmann C, Lefort T, Pialat JB. Ultrasound shear wave velocity in skeletal muscle: A reproducibility study. Diagn Interv Imaging 2016;97:71-79.

19. Ateş F, Hug F, Bouillard K, et al. Muscle shear elastic modulus is linearly related to muscle torque over the entire range of isometric contraction intensity. J Electromyogr Kinesiol 2015;25:703-708.

20. Song P, Macdonald MC, Behler RH, et al. Shear wave elastography on the GE LOGIQ E9 with Comb-push U1trasound Shear Elastography (CUSE) and time aligned sequential tracking (TAST). In: IEEE International Ultrasonics Symposium 2014:1101-1104.. IEEE Computer Society. doi:10.1109/ULTSYM.2014.0270.

21. Bercoff J, Tanter M, Fink M. Supersonic shear imaging: a new technique for soft tissue elasticity mapping. IEEE Trans Ultrason Ferroelectr Freq Control 2004;51:396-409.

22. Bland JM, Altman DG. Measurement error. BMJ 1996;313:744.
23. Bland JM, Altman DG. Measurement error proportional to the mean. BMJ 1996;313:106.

24. Landis JR, Koch GG. The measurement of observer agreement for categorical data. Biometrics 1977;33:159-174.

25. Eliasziw M, Young SL, Woodbury MG, Fryday-Field K. Statistical methodology for the concurrent assessment of interrater and intrarater reliability: using goniometric measurements as an example. Phys Ther 1994;74:777-788.

26. Bland JM, Altman DG. Measuring agreement in method comparison studies. Stat Methods Med Res 1999;8:135160 .

27. Nordez A, Gennisson JL, Casari P, Catheline S, Cornu C. Characterization of muscle belly elastic properties during passive stretching using transient elastography. J Biomech 2008;41:2305-2311.

28. Shinohara M, Sabra K, Gennisson JL, Fink M, Tanter M. Real-time visualization of muscle stiffness distribution with ultrasound shear wave imaging during muscle contraction. Muscle Nerve 2010;42:438-441.

29. Levinson SF, Shinagawa M, Sato T. Sonoelastic determination of human skeletal muscle elasticity. J Biomech 1995;28:1145-1154.

30. Carpenter EL, Lau HA, Kolodny EH, Adler RS. Skeletal Muscle in Healthy Subjects versus Those with GNE-Related Myopathy: Evaluation with Shear-Wave US--A Pilot Study. Radiology 2015;277:546-554.

31. Lacourpaille L, Hug F, Bouillard K, Hogrel JY, Nordez A. Supersonic shear imaging provides a reliable measurement of resting muscle shear elastic modulus. Physiol Meas 2012;33:N19-N28.

32. Song P, Macdonald MC, Behler RH, et al. Two-dimensional shear-wave elastography on conventional ultrasound scanners with time-aligned sequential tracking (TAST) and comb-push ultrasound shear elastography (CUSE). IEEE Trans Ultrason Ferroelectr Freq Control 2015;62:290-302. 